\title{
Collective photon routing improvement in a dissipative quantum emitter chain strongly coupled to a chiral waveguide QED ladder
}

\author{
Bibandhan Poudyal and Imran M. Mirza $\odot^{*}$ \\ Macklin Quantum Information Sciences, Department of Physics, Miami University, Oxford, Ohio 45056, USA
}

(Received 20 June 2020; accepted 18 September 2020; published 8 October 2020)

\begin{abstract}
We examine the routing scheme of single photons in a one-dimensional periodic chain of two-level quantum emitters (QEs) strongly coupled to two waveguides in a ladder configuration. It is known that for a single-emitter chiral waveguide ladder setting photons can be redirected from one waveguide to another with a $100 \%$ probability (deterministically) provided the resonance condition is met and spontaneous emission is completely ignored. However, when the spontaneous emission is included the routing scheme becomes considerably imperfect. In this paper, we present a solution to this issue by considering a chain of QEs where, in addition to the waveguide mediated interaction among emitters, a direct and infinitely long-ranged dipole-dipole interaction (DDI) is taken into account. We show that the collective effects arising from the strong DDI protect the routing scheme from spontaneous emission loss. In particular, we demonstrate that the router operation can be improved from 58 to $\approx 95 \%$ in a typical dissipative chiral light-matter interface consisting of nanowire modes strongly interacting with a linear chain of 30 quantum dots. With the recent experimental progress in chiral quantum optics, trapped QEs evanescently coupled to tapered nanofibers can serve as a platform for the experimental realization of this paper.
\end{abstract}

DOI: 10.1103/PhysRevResearch.2.043048

\section{INTRODUCTION}

Several quantum networking protocols rely on using single photons as the carrier of quantum information as they propagate quickly and can cover long distances without being considerably influenced by decoherence effects [1]. Typically, stationary qubits [quantum emitters (QEs)] are placed at the nodes in these networks to store, manipulate, and retrieve the quantum information while the photons, guided by optical fibers, transfer information from one node to another. Switching or routing of single photons turns out to be a key requirement in such networks to interlink different nodes $[2,3]$. For a reliable interlinking protocol, the routing scheme is required to be not only efficient but also able to preserve the quantum state of the photons initially launched into the network.

Historically, Harris and Yamamoto were the first to propose, in 1998, that quantum interference can be utilized for photon switching in a four-level atomic system [4]. In the last decade or so, several proposals have been put forward and experiments have been performed to accomplish efficient photon routing. For instance, it has been theoretically proposed that electromagnetically induced transparency (EIT) in cavity optomechanics can be exploited for weak probe field

\footnotetext{
*mirzaim@miamioh.edu

Published by the American Physical Society under the terms of the Creative Commons Attribution 4.0 International license. Further distribution of this work must maintain attribution to the author(s) and the published article's title, journal citation, and DOI.
}

photon switching [5]. There has also been a series of routing proposals reported which are based on QEs embedded in coupled resonator waveguides (Refs. [6-10] to name a few). In other proposals circuit quantum electrodynamics QED systems have been used to attain routing through EIT (see, for example, Ref. [11]). On the experimental side, photon switching or routing has also been demonstrated in cavity QED setups $[12,13]$ and in the microwave domain using artificial atoms (such as transmon qubits) interacting with transmission lines [14].

Waveguide QED architectures are particularly appealing in this regard as these structures can be used to perform several quantum information tasks in a single setup [15] including photon routing [16-18]. Closely akin to waveguide QED is the burgeoning development of chiral quantum optical systems [19]. Chiral atom-field interaction here refers to propagationdirection-dependent absorption/emission of light which is achieved by the transverse confinement of electromagnetic radiation in subwavelength diameter optical fibers. It is known that such confinement leads to the spin-momentum locking of light [20]. One interesting consequence of this effect is that, if we bring a QE close to such a fiber, then if the polarization of light is matched with the polarization-dependent absorption of the QE the light propagation is locked with the absorption properties of the emitter. As a result, light propagating in a certain direction in the fiber can be absorbed by such an emitter but the light propagating in the opposite direction fails to be absorbed. Typically the waveguides used in these types of experiments are nearly lossless and the evanescent field dies off exponentially; therefore, once the photon is emitted back into such chiral fiber modes it is not lost. Since 2012, several experiments have been performed on the subject of chiral 


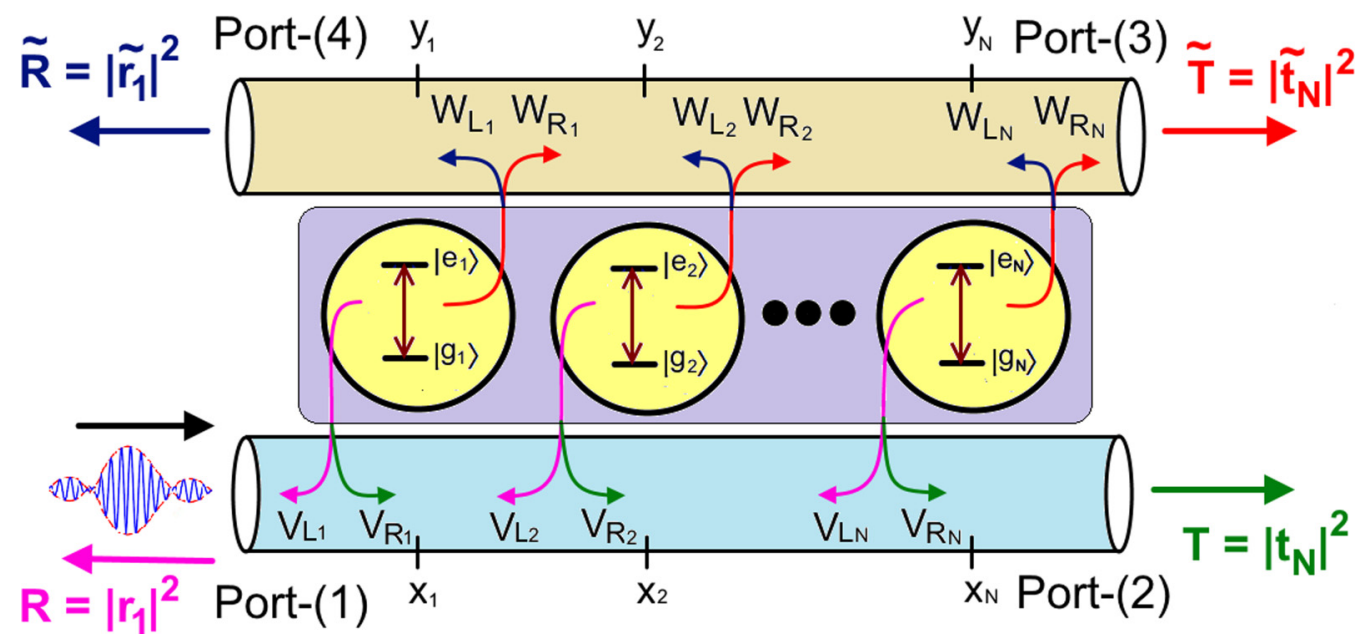

FIG. 1. Illustration of a periodic multiemitter chain coupled to a double waveguide QED system. A single-photon wave packet is launched from port 1 and we are interested in the port 1 to port 3 routing scheme. The purple-colored region (which should not be confused with a substrate) here shows the presence of infinitely long-range dipole-dipole interaction among all QEs. Infinitely long-ranged interaction means that each emitter is coupled with every other emitter in the chain. $R, T, \widetilde{R}$, and $\widetilde{T}$ represent intensities of reflection from the bottom waveguide, transmission from the bottom waveguide, leftward rectification from the upper waveguide, and rightward rectification from the upper waveguide, respectively. In the experimental realization of such a one-dimensional chain of emitters, Rydberg atom architectures can be used as a possible platform [15]. Therein, with the aid of tight optical traps (tweezers), single emitters can be loaded into each trapping site and a one-dimensional chain of emitters can be realized. Furthermore, such a Rydberg emitter chain can be coupled with microwave coplanar waveguides as discussed in detail in Ref. [15].

quantum optics with many exciting applications [21-28]. In 2015 , more than $90 \%$ directionality and $98 \%$ atom-waveguide coupling strength have been reported in photonic crystal waveguides [29].

In this paper, we analyze the nonreciprocal routing of single photons in many-emitter chiral waveguide QED in a ladder geometry. In such a four-port device, it is known that for a single-emitter case perfect/deterministic routing can be achieved if the resonant interaction between the emitter and chiral waveguides is ensured in the absence of spontaneous emission (see, for example, Cheng et al. [30], GonzalezBallestero et al. [31], and Yan et al. [32]). However, in a more realistic scenario in which a finite spontaneous emission is included, routing efficiency is considerably impacted and shows a drastic reduction. Herein, we focus on this issue and discuss how the routing scheme can be notably improved in the presence of spontaneous emission loss. To this end, we consider a one-dimensional chain of two-level QEs strongly coupled to the waveguide ladder setting. In our model, we further assume that in addition to waveguide mediated indirect interactions among QEs there also exists an infinitely longranged direct dipole-dipole interaction (DDI). To meet the DDI condition we suppose the interemitter separation is less than a single resonant wavelength [33].

For the case of two dissipative QEs strongly coupled to both chiral waveguides, we analytically find that the output spectra split into a frequency doublet due to waveguide mediated interaction without DDI. The inclusion of DDI introduces an asymmetry in the peak heights which can be exploited to improve the routing efficiency in the presence of dissipation (spontaneous emission). Furthermore, we numerically extend our results to the many-emitter regime. Therein, we demonstrate that for a chain of 30 identical QEs strongly coupled through waveguide modes as well as through DDI, with an interaction rate $\Gamma=11.03 \Gamma_{0}\left(\Gamma_{0}\right.$ being the free space decay rate), the routing efficiency can be improved from 58 to $\approx 95 \%$ with a DDI of strength $\approx 2.1 \Gamma$ in the presence of a spontaneous emission rate of $\approx 0.62 \Gamma$.

The paper is organized as follows. In Sec. II we describe the system model. In Sec. III we discuss the single-photon transport theory in a many-emitter waveguide QED ladder. Section IV presents the results, mainly focusing on the issue of how collective effects can promote routing efficiency. Finally, in Sec. V we summarize the main conclusions of this paper.

\section{MODEL AND HAMILTONIAN}

As shown in Fig. 1, we consider a periodic array of two-level QEs (herein also referred to as qubits/atoms) simultaneously side coupled to two one-dimensional waveguides forming a double-waveguide multiemitter QED ladder. The total Hamiltonian of the system can be decomposed into four pieces:

$$
\hat{\mathscr{H}}=\hat{\mathcal{H}}_{2 \mathrm{LS}}+\hat{\mathcal{H}}_{\mathrm{wav}}+\hat{\mathcal{H}}_{\text {int }}+\hat{\mathcal{H}}_{\mathrm{DDI}} .
$$

$\hat{\mathcal{H}}_{2 \text { LS }}$ describes the free Hamiltonian of $N$ number of two-level systems (2LS) or qubits with the $j$ th qubit ground state given by $\left|g_{j}\right\rangle$ and the excited state given by $\left|e_{j}\right\rangle$. The absolute frequencies of these states are given by $\omega_{g_{j}}$ and $\omega_{e_{j}}$, respectively. Here $j \in\{1, N\}$ and $\widetilde{\omega}_{e_{j}} \equiv \omega_{e_{j}}-i \gamma_{j} / 2$, where $\gamma_{j}$ represents the phenomenologically added spontaneous emission rate of the $j$ th emitter. The atomic raising $\hat{\sigma}_{j}^{\dagger}$ and lowering operator $\hat{\sigma}_{j}$ follow the standard Fermionic anticommutation relation 
$\left\{\hat{\sigma}_{j}^{\dagger}, \hat{\sigma}_{k}\right\}=\delta_{j k}$. With these specifications, $\hat{\mathcal{H}}_{2 \mathrm{LS}}$ takes the form

$$
\begin{aligned}
\hat{\mathcal{H}}_{2 \mathrm{LS}} & =\sum_{j=1}^{N}\left(\hbar \widetilde{\omega}_{e_{j}}\left|e_{j}\right\rangle\left\langle e_{j}\left|+\hbar \omega_{g_{j}}\right| g_{j}\right\rangle\left\langle e_{j}\right|\right) \\
& \equiv \hbar \sum_{j=1}^{N}\left(\widetilde{\omega}_{e_{j}} \hat{\sigma}_{j}^{\dagger} \hat{\sigma}_{j}+\omega_{g_{j}} \hat{\sigma}_{j} \hat{\sigma}_{j}^{\dagger}\right) .
\end{aligned}
$$

The free-field Hamiltonian $\hat{\mathcal{H}}_{\text {wav }}$ incorporates the optical modes of two bidirectional waveguides. In the realspace formalism of quantum optics [34], $\hat{c}_{\alpha}(x) / \hat{c}_{\alpha}^{\dagger}(x)$ and $\hat{b}_{\alpha}(y) / \hat{b}_{\alpha}^{\dagger}(y)$ [for $\alpha=L$ (left) and $R$ (right)] represent field annihilation/creation operators in the downward and upward waveguides, respectively. These operators follow the usual bosonic commutation relations $\left[\hat{c}_{\alpha}(x), \hat{c}_{\beta}^{\dagger}\left(x^{\prime}\right)\right]=\delta_{\alpha \beta} \delta\left(x-x^{\prime}\right)$ and $\left[\hat{b}_{\alpha}(y), \hat{b}_{\beta}^{\dagger}\left(y^{\prime}\right)\right]=\delta_{\alpha \beta} \delta\left(y-y^{\prime}\right)$ where $\beta=L, R$. If we assume the group velocity of the field to be $v_{g_{d}}\left(v_{g_{u}}\right)$ in the downward (upward) waveguide then in the linearized dispersion regime $\hat{\mathcal{H}}_{\text {wav }}$ can be expressed as

$$
\begin{aligned}
\hat{\mathcal{H}}_{\mathrm{wav}}= & -i \hbar v_{g_{d}} \int d x\left[\hat{c}_{R}^{\dagger}(x) \partial_{x} \hat{c}_{R}(x)-\hat{c}_{L}^{\dagger}(x) \partial_{x} \hat{c}_{L}(x)\right] \\
& -i \hbar v_{g_{u}} \int d y\left[\hat{b}_{R}^{\dagger}(y) \partial_{y} \hat{b}_{R}(y)-\hat{b}_{L}^{\dagger}(y) \partial_{y} \hat{b}_{L}(y)\right] .
\end{aligned}
$$

$\hat{\mathcal{H}}_{\text {int }}$ represents the atom-field interaction Hamiltonian under the rotating wave approximation. Expressing the interaction strength between emitters and the upward waveguide (emitters and the lower waveguide) by real-valued parameters $V_{\alpha_{j}}\left(W_{\alpha_{j}}\right)$ and specifying emitter locations by the presence of Dirac delta functions on field variables, we write

$$
\begin{aligned}
\hat{\mathcal{H}}_{\text {int }}= & \hbar \sum_{j=1}^{N} \sum_{\alpha=L, R}\left\{\int d x \delta\left(x-x_{j}\right)\left[V_{\alpha_{j}} \hat{c}_{\alpha}^{\dagger}(x) \hat{\sigma}_{j}+\text { H.c. }\right]\right. \\
& \left.+\int d y \delta\left(y-y_{j}\right)\left[W_{\alpha_{j}} \hat{b}_{\alpha}^{\dagger}(y) \hat{\sigma}_{j}+\text { H.c. }\right]\right\} .
\end{aligned}
$$

Finally, we assume that the separation between the atoms is smaller than the wavelength of the resonant field. This means that in addition to being indirectly coupled through waveguide-mediated interactions the emitters can also interact directly through infinitely long-ranged dipole-dipole interactions [35]. With this consideration, the DDI part of the Hamiltonian is given by

$$
\hat{\mathcal{H}}_{\mathrm{DDI}}=\hbar \sum_{i=1}^{N} \sum_{\substack{j=1 \\ j>i}}^{N} J_{i j}\left(\hat{\sigma}_{i}^{\dagger} \hat{\sigma}_{j}+\text { H.c. }\right) \text {. }
$$

The strength of the DDI $J_{i j} \equiv J\left(\mathcal{R}_{i j}\right)$ sensitively depends on the interemitter separation $\mathcal{R}_{i j}$ and is expressed as [36]

$$
\begin{aligned}
J\left(\mathcal{R}_{i j}\right)= & \frac{3 \Gamma_{0}}{4}\left(\frac{\cos \mathcal{R}_{i j}}{\mathcal{R}_{i j}^{3}}+\frac{\sin \mathcal{R}_{i j}}{\mathcal{R}_{i j}^{2}}-\frac{\cos \mathcal{R}_{i j}}{\mathcal{R}_{i j}}\right) \\
& +\cos ^{2} \theta\left(\frac{\cos \mathcal{R}_{i j}}{\mathcal{R}_{i j}}-\frac{3 \cos \mathcal{R}_{i j}}{\mathcal{R}_{i j}^{3}}-\frac{3 \sin \mathcal{R}_{i j}}{\mathcal{R}_{i j}^{2}}\right) .
\end{aligned}
$$

Here $\mathcal{R}_{i j}=\omega_{e g}\left|\vec{r}_{i}-\vec{r}_{j}\right| / c, c$ is the speed of light, $\omega_{e g}=\omega_{e}-$ $\omega_{g}, \vec{r}_{i / j}$ gives the location of the $i$ th $/ j$ th emitter, and $\Gamma_{0}$ is the free space decay rate of the emitter (also used as the unit in this paper). $\theta$ is the angle between the dipole moment $\vec{p}$ of the emitters and the position vector which is defined by $\cos \theta=$ $\vec{p} \cdot\left(\vec{r}_{i}-\vec{r}_{j}\right) /\left\{|\vec{p}|\left|\vec{r}_{i}-\vec{r}_{j}\right|\right\}$.

\section{PHOTON TRANSPORT THEORY}

To investigate the scattering of single photons, the eigenstate $|\Psi\rangle$ of the Hamiltonian $\hat{\mathscr{H}}$ in the single excitation manifold of the combined Hilbert space can be constructed as

$$
\begin{aligned}
|\Psi\rangle= & {\left[\sum_{j=1}^{N} \mathcal{A}_{j} \hat{\sigma}_{j}^{\dagger}+\sum_{\alpha=L, R}\left\{\int d x \varphi_{\alpha}(x) \hat{c}_{\alpha}^{\dagger}(x)\right.\right.} \\
& \left.\left.+\int d y \chi_{\alpha} \hat{b}_{\alpha}^{\dagger}(y)\right\}\right]|\varnothing\rangle,
\end{aligned}
$$

where $|\varnothing\rangle=\left|g_{1}, g_{2}, \ldots, g_{N}\right\rangle \otimes\left|0_{d R}, 0_{d L}\right\rangle \otimes\left|0_{u R}, 0_{u L}\right\rangle$ is the ground state of the combined system where all atoms are unexcited and there are zero photons in the upper $(u)$ and downward $(d)$ waveguides in both left and right directions. $\mathcal{A}_{j}, \varphi_{\alpha}$, and $\chi_{\alpha}$, respectively, denote the probability amplitudes of finding the $j$ th emitter in the excited-state photon to be in the bottom and upper waveguide in the $\alpha$ th direction. Initially, we suppose that there are no photons in the waveguides, and all QEs are unexcited. For the single-photon routing problem under study, without loss of generality, we assume that the single photon is launched from port 1 and we are interested in the port 1 to port 3 nonreciprocal routing scheme.

The steady-state analysis of the problem requires the diagonalization of the Hamiltonian $\mathscr{H}$. To this end, we insert Eqs. (1) and (7) in the time-independent Schrödinger equation: $\hat{\mathscr{H}}|\Psi\rangle=\hbar \omega|\Psi\rangle$, where $\hbar \omega$ is the energy of the input photon. We obtain the following set of ordinary differential equations for the required probability amplitudes:

$$
\begin{aligned}
-i v_{g_{D}} \frac{\partial \varphi_{\alpha}(x)}{\partial x}+\sum_{j=1}^{N} V_{\alpha_{j}} \mathcal{A}_{j} \delta\left(x-x_{j}\right) & =\omega \varphi_{\alpha}(x), \\
-i v_{g_{D}} \frac{\partial \chi_{\alpha}(y)}{\partial y}+\sum_{j=1}^{N} W_{\alpha_{j}} \mathcal{A}_{j} \delta\left(y-y_{j}\right) & =\omega \chi_{\alpha}(y), \\
\sum_{\alpha=L, R}\left[V_{\alpha_{j}} \varphi_{\alpha}\left(x_{j}\right)+W_{\alpha_{j}} \chi_{\alpha}\left(y_{j}\right)\right] & =\left(\omega-\widetilde{\omega}_{e g_{j}}\right) \mathcal{A}_{j} \\
& -\sum_{\substack{i=1 \\
i \neq j}}^{N} J_{i j} \mathcal{A}_{i} .
\end{aligned}
$$

Here, for $\alpha=R, D=d$ and, for $\alpha=L, D=u$. In the above equation set, we notice that the DDI interaction appears in the last equation and gives rise to infinitely long-range coupling among all QEs. To solve the equation set (8) we integrate the first equation from $x_{k}-\epsilon$ to $x_{k}+\epsilon$ and the next equation from $y_{k}-\epsilon$ to $y_{k}+\epsilon . x_{k}\left(y_{k}\right)$ represents the location of the $k$ th 
emitter for the downward (upward) waveguide and $\epsilon \ll 1$. As a result, we find the following four discontinuity conditions:

$$
\begin{aligned}
& \varphi_{\alpha}\left(x_{j}+\epsilon\right)-\varphi_{\alpha}\left(x_{j}-\epsilon\right)=\frac{-i V_{\alpha_{j}}}{v_{g_{D}}} \mathcal{A}_{j}, \\
& \chi_{\alpha}\left(y_{j}+\epsilon\right)-\chi_{\alpha}\left(y_{j}-\epsilon\right)=\frac{-i W_{\alpha_{j}}}{v_{g_{D}}} \mathcal{A}_{j} .
\end{aligned}
$$

Afterward, we establish a connection between $\varphi_{\alpha}(x)$ and $\varphi_{\alpha}\left(x_{j} \pm \epsilon\right)$, and similarly between $\chi_{\alpha}(y)$ and $\chi_{\alpha}\left(y_{j} \pm \epsilon\right)$, by applying the following regularization relationships:

$$
\begin{aligned}
& \varphi_{\alpha}\left(x_{j}\right)=\lim _{\epsilon \rightarrow 0}\left[\frac{\varphi_{\alpha}\left(x_{j}+\epsilon\right)+\varphi_{\alpha}\left(x_{j}-\epsilon\right)}{2}\right], \\
& \chi_{\alpha}\left(y_{j}\right)=\lim _{\epsilon \rightarrow 0}\left[\frac{\chi_{\alpha}\left(y_{j}+\epsilon\right)+\chi_{\alpha}\left(y_{j}-\epsilon\right)}{2}\right] .
\end{aligned}
$$

Next, we assume plane-wave solutions for the field amplitudes that are modified at each boundary (location of an emitter) by respective transmission, reflection, rightward rectification, and leftward rectification amplitudes. Such an ansatz engenders the following solution:

$$
\begin{aligned}
& \varphi_{R}(x)=\left\{\begin{array}{ll}
t_{0} e^{i q x}, & x<x_{1} \\
t_{1} e^{i q x}, & x_{1} \leqslant x \leqslant x_{2} \\
\vdots & \\
t_{N} e^{i q x}, & x>x_{N}
\end{array},\right. \\
& \varphi_{L}(x)=\left\{\begin{array}{l}
r_{1} e^{-i q x}, \quad x<x_{1} \\
r_{2} e^{-i q x}, \quad x_{1} \leqslant x \leqslant x_{2} \\
\vdots \\
r_{N+1} e^{-i q x}, \quad x>x_{N}
\end{array},\right. \\
& \chi_{R}(y)=\left\{\begin{array}{l}
\widetilde{t}_{0} e^{i m y}, \quad y<y_{1} \\
\widetilde{t}_{1} e^{i m y}, \quad y_{1} \leqslant y \leqslant y_{2} \\
\vdots \\
\tilde{t}_{N} e^{i m y}, \quad y>y_{N}
\end{array}\right. \\
& \chi_{L}(y)=\left\{\begin{array}{l}
\widetilde{r}_{1} e^{-i m y}, \quad y<y_{1} \\
\widetilde{r}_{2} e^{-i m y}, \quad y_{1} \leqslant y \leqslant y_{2} \\
\vdots \\
\widetilde{r}_{N+1} e^{-i m y}, \quad y>y_{N}
\end{array} .\right.
\end{aligned}
$$

In our model single photons are launched from port 1 and there are no other inputs; therefore, we fix $t_{0}=1, r_{N+1}=$ $0, \widetilde{t}_{0}=0$, and $\widetilde{r}_{N+1}=0$. For a chain of identical emitters the optical wave numbers $q$ and $m$ are defined through $q=(\omega-$ $\left.\omega_{e g}\right) / v_{g_{d}}$ and $m=\left(\omega-\omega_{e g}\right) / v_{g_{u}}$, respectively. The relation among various amplitudes can be found by inserting Eq. (11) into Eqs. (9) and (8), which after simplifications produce the following set of recurrence relations:

$$
\begin{aligned}
& t_{j}-t_{j-1}=\frac{-i V_{R_{j}}}{v_{g_{d}}} \mathcal{A}_{j} e^{-i q x_{j}}, \\
& r_{j+1}-r_{j}=\frac{i V_{L_{j}}}{v_{g_{d}}} \mathcal{A}_{j} e^{i q x_{j}}, \\
& \tilde{t}_{j}-\tilde{t}_{j-1}=\frac{-i W_{R_{j}}}{v_{g_{u}}} \mathcal{A}_{j} e^{-i m y_{j}}, \\
& \widetilde{r}_{j+1}-\widetilde{r}_{j}=\frac{i W_{L_{j}}}{v_{g_{u}}} \mathcal{A}_{j} e^{i m y_{j}},
\end{aligned}
$$

$$
\begin{gathered}
\frac{V_{R_{j}}}{2} e^{i q x_{j}}\left(t_{j}+t_{j-1}\right)+\frac{V_{L_{j}}}{2} e^{-i q x_{j}}\left(r_{j+1}+r_{j}\right) \\
+\frac{W_{R_{j}}}{2} e^{i m y_{j}}\left(\widetilde{t}_{j}+\widetilde{t}_{j-1}\right)+\frac{W_{L_{j}}}{2} e^{-i m y_{j}}\left(\widetilde{r}_{j+1}+\widetilde{r}_{j}\right) \\
=\left(\omega-\widetilde{\omega}_{e g_{j}}\right) \mathcal{A}_{j}-\sum_{\substack{i=1 \\
i \neq j}}^{N} J_{i j} \mathcal{A}_{i} .
\end{gathered}
$$

The required net transmission, reflection, rightward rectification, and leftward rectification intensities (which can be calculated by solving the above set of coupled equations for any $j$ value) are defined by $T=\left|t_{N} e^{i q N L}\right|^{2}, R=\left|r_{1}\right|^{2}$, $\widetilde{T}=\left|\widetilde{t}_{N} e^{i m N L}\right|^{2}$, and $\widetilde{R}=\left|\widetilde{r}_{1}\right|^{2}$, respectively. It is worthwhile to mention that the time-delay (retardation) effects are already incorporated in our model due to the presence of propagation phases $e^{i q x_{j}}$ and $e^{i m y_{j}}$. For chiral cases, these phases do not appear in the output intensities because of the unidirectionality of the problem. However, when the direct back reflections between QEs are added due to DDI then these retardation phases impact the photon transport.

\section{RESULTS}

There are various experimental platforms where the results of our generic theoretical model can be applied. Examples include superconducting Josephson junctions (artificial atoms) in microwave transmission lines [37], cesium atoms coupled to photonic crystal waveguides [38], and semiconducting quantum dots (QDs) interacting with nanowires (nanofibers) [39]. Following experimental setups studied in Refs. [36,39,40], we consider a chain of QDs interacting with two identical Ag nanowires. The transition wavelength $\lambda_{\mathrm{QD}}$ is taken to be $655 \mathrm{~nm}$ with spontaneous emission rate $\gamma=6.86 \Gamma_{0}$ and emitter-waveguide coupling strength $\Gamma=$ $11.03 \Gamma_{0}$. The free space decay rate $\Gamma_{0}$ is allotted a value of $7.5 \mathrm{MHz}$.

The interemitter separation appears at two places in our calculations: (I) in the expression of DDI and (II) in the free propagation phases $\left(q x_{j}\right.$ and $\left.m y_{j}\right)$ accounting for the retardation effects. In the DDI expression, for the sake of simplicity, we assume the dipole moment of all QDs to be perpendicular to the position vector direction implying $\cos \theta=0$. For any two consecutive emitters at positions $(0,17 \mathrm{~nm}, 0)$ and (32.75 nm, $17 \mathrm{~nm}, 0)$ (lattice constant of $32.75 \mathrm{~nm}$ ), from Eq. (6) the DDI interaction turns out to be $J_{12}=J_{21} \equiv J=$ $23.10 \Gamma_{0}$. For the free propagation phase, we consider the wavelength of the surface plasmon $\lambda_{\mathrm{SP}}$ to be $211.8 \mathrm{~nm}<\lambda_{\mathrm{QD}}$ due to a reduction in the group velocity in the waveguide. If we assume both waveguides to be identical then these phases take the value $q x_{j}=m y_{j} \equiv \Theta=0.31 \pi$.

\section{A. Routing with a single emitter}

Let us begin with the simplest case of a single emitter. In the following, we focus on two scenarios, namely, symmetric coupling and chiral coupling cases. 

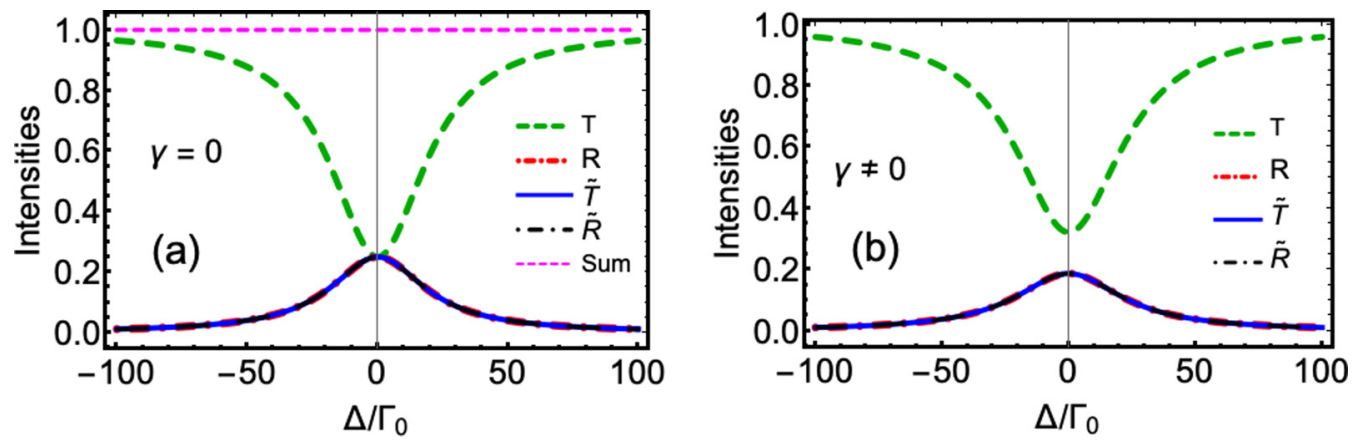

FIG. 2. Transport intensities vs detuning under the symmetric coupling scenario. For plot (a) we ignore the spontaneous emission, i.e., $\gamma=0$, while in plot (b) $\gamma \neq 0$. The emitter-waveguide coupling strength is fixed to $\Gamma=11.03 \Gamma_{0}$ while the spontaneous emission rate $\gamma$ is $6.86 \Gamma_{0}$.

\section{Symmetric coupling case}

In the symmetric case, we take the same value of emitterwaveguide coupling towards left and right, i.e., $V_{R}=V_{L}=$ $W_{R}=W_{L} \equiv \mathcal{U}$ and $v_{g_{d}}=v_{g_{u}} \equiv v_{g}$ such that $\Gamma=\mathcal{U}^{2} / v_{g}$. The parameter $\Gamma$ is defined to quantify emitter-waveguide coupling strength. One can find the transport amplitudes by inserting $j=1$ in Eq. (12) and solve for $t_{1} \equiv t, r_{1} \equiv r, \widetilde{t_{1}} \equiv \widetilde{t}$, and $\widetilde{r}_{1} \equiv \widetilde{r}$. We obtain

$$
\begin{aligned}
& t=\frac{-8 \Gamma^{3}-4 \Gamma^{2}(\gamma-2 i \Delta)+2 \Gamma(\gamma-2 i \Delta)^{2}+(\gamma-2 i \Delta)^{3}}{(\gamma-2 \Gamma-2 i \Delta)(\gamma+2 \Gamma-2 i \Delta)(\gamma+4 \Gamma-2 i \Delta)}, \\
& r=\tilde{t}=\tilde{r}=\left(\frac{-2 \Gamma(2 \gamma-2 i \Delta)+4 \Delta^{2}-\gamma^{2}+4 i(\gamma+\Gamma) \Delta}{2 \Gamma(\gamma-2 i \Delta)}\right)^{-1} .
\end{aligned}
$$

In Fig. 2(a), we plot probabilities of detecting the photon at different output ports as a function of detuning $\Delta \equiv \omega-\omega_{e g}$. For this plot, we set $\gamma=0$. As expected we find $T+R+$ $\widetilde{T}+\widetilde{R}=1$ ensuring proper normalization. More importantly, from Eq. (13) we notice that at $\Delta=0$ all intensities share a common value of 0.25 which indicates an equal probability of detecting the photon at all four ports (as also reported in Refs. [6,32]). Such behavior is completely contrary to the required routing protocol where the photon is expected to emerge at port 3 with $100 \%$ probability. Under far-off resonance conditions, we find that the transmission into port 2 reaches almost $100 \%$ at the expense of decreasing intensities at other ports. A complete mismatch between incoming photon frequency and $\omega_{e g}$ results in such behavior where the photon continues to propagate in the bottom waveguide where eventually it is detected at port 2 without being routed.

In Fig. 2(b), we introduce a finite spontaneous emission rate. With $\gamma=6.86 \Gamma_{0}$, we notice that the on-resonance output intensities $T=\widetilde{T}=\widetilde{R}$ decrease to $\approx 19 \%$ while the reflection intensity from the bottom waveguide $\widetilde{R}$ raises to $\approx 32 \%$. The remaining probability contribution is lost to nonwaveguide (environmental) modes. From Figs. 2(a) and 2(b) we conclude that symmetric coupling fails to attain deterministic routing. But what would happen if we could break the symmetry in QE emission into the waveguide modes? Next, we answer this question by taking advantage of the chiral photon emissions.

\section{Chiral coupling case}

For the chiral case, we suppose that the QE is allowed to interact only with the right propagating modes in both waveguides with back-reflection channels completely blocked, i.e., we set $\Gamma_{d L} \equiv V_{L}^{2} / v_{g_{d}}=0, \Gamma_{u L} \equiv W_{L}^{2} / v_{g_{u}}=0$ with $\Gamma_{d R}=\Gamma_{u R}=\Gamma$ while $\Gamma_{d R} \equiv V_{R}^{2} / v_{g_{d}}$ and $\Gamma_{u R} \equiv W_{R}^{2} / v_{g_{u}}$. Under these conditions, the transport amplitudes take the form

$$
\begin{array}{ll}
t=\left(\frac{\gamma-2 i \Delta}{\gamma+2 \Gamma-2 i \Delta}\right), & r=0 ; \\
\tilde{t}=\left(\frac{-2 \Gamma}{\gamma+2 \Gamma-2 i \Delta}\right), & \tilde{r}=0 .
\end{array}
$$

In Fig. 3, we plot the output intensities for such a chiral setting. Figure 3(a) concentrates on the no-loss situation. We note that at $\Delta=0$ the transmission from the bottom waveguide $T$ vanishes. As pointed out in Refs. [31,34], such behavior is attributed to the perfect destructive interference between the amplitudes of incoming photons and the photons emitted into the forward direction of the bottom waveguide. Consequently, the rightward rectification $\widetilde{T}$ takes a unit value with the photon emerging at port 3 acquiring a phase shift of $\pi$. Thus we conclude that chiral emitter-waveguide couplings can be used for perfect photorouting if the conditions of on-resonance and $\gamma=0$ are met.

However, in any realistic experimental proposal spontaneous emission cannot be ignored. With this concern, in Fig. 3(b) we present intensities with $\gamma \neq 0$. From Eq. (14), we find the transport amplitudes take the values $t=(1+$ $2 \Gamma / \gamma)^{-1}$ and $\tilde{t}=-(1+0.5 \gamma / \Gamma)^{-1}$ at resonance. We notice that, with a $\gamma$ value of about $62 \%$ of the emitter-waveguide coupling rate, even in the presence of chirality and perfect resonance conditions, the efficiency of the routing scheme drastically reduces to $58 \%$. Additionally, $T$ raises 

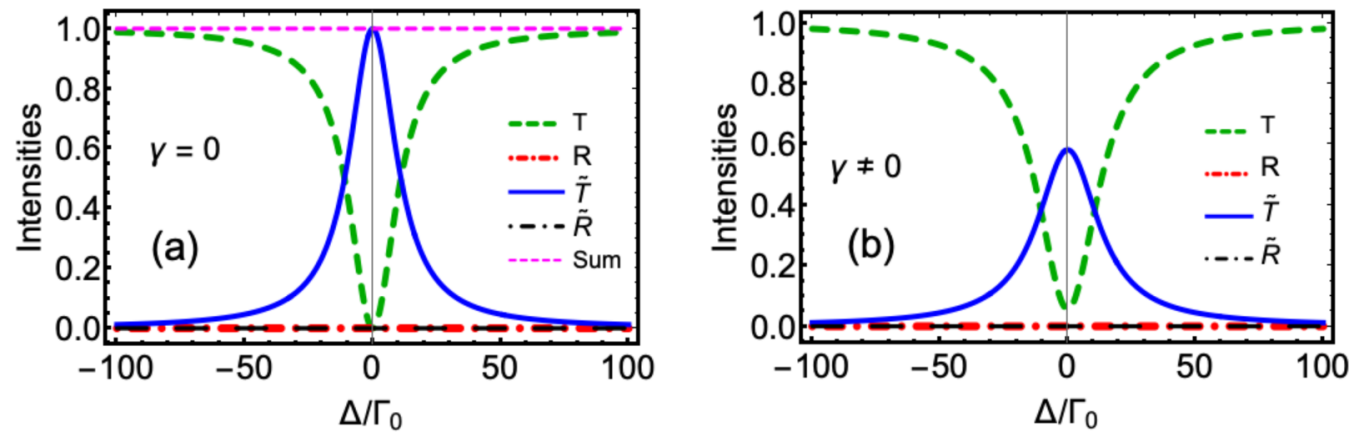

FIG. 3. Port intensities as a function of detuning for a perfect chiral situation in which photon emission in the left direction is not allowed: (a) $\gamma=0$ and (b) $\gamma \neq 0$. In this plot $\Gamma=11.03 \Gamma_{0}$ and $\gamma=6.86 \Gamma_{0}$.

to $\approx 6 \%$. From this result, it is evident that the deterministic nature of the routing scheme achieved by chirality is lost when QEs are allowed to spontaneously emit the photons.

\section{B. Routing with collective response of two QEs}

We now concentrate on the question, which is central to this paper, of in what ways the routing of photons can be improved in the presence of dissipative loss from QEs chirally coupled to a waveguide ladder. To this end, we first analyze the routing in the presence of two QEs simultaneously interacting through waveguide modes and DDI. From Eq. (12) we obtain the analytic expressions for net transmission $t_{2}$ and rightward rectification amplitude $\widetilde{t}_{2}$ as

$$
\begin{aligned}
& t_{2}=\frac{-2 i \sin \Theta J \Gamma+i\left(4 J^{2}+\gamma^{2}+4 \Gamma^{2}-4 i \gamma \Delta-4 \Delta^{2}\right)}{4 i J^{2}+8 e^{i \Theta} J \Gamma+i(\gamma+2 \Gamma-2 i \Delta)^{2}} \\
& \tilde{t}_{2}=\frac{-4 e^{-i \Theta} J \Gamma\left(1+e^{2 i \Theta}\right)-4 \Gamma(i \gamma+2 \Delta)}{4 i J^{2}+8 e^{i \Theta} J \Gamma+i(\gamma+2 \Gamma-2 i \Delta)^{2}}
\end{aligned}
$$

In Fig. 4 we present the probabilities of photon detection at all four ports. In these plots, we mainly concentrate on two parameters, DDI $(J)$ and spontaneous emission rate $(\gamma)$. In Fig. 4(a) we present the simplest case when both QEs are
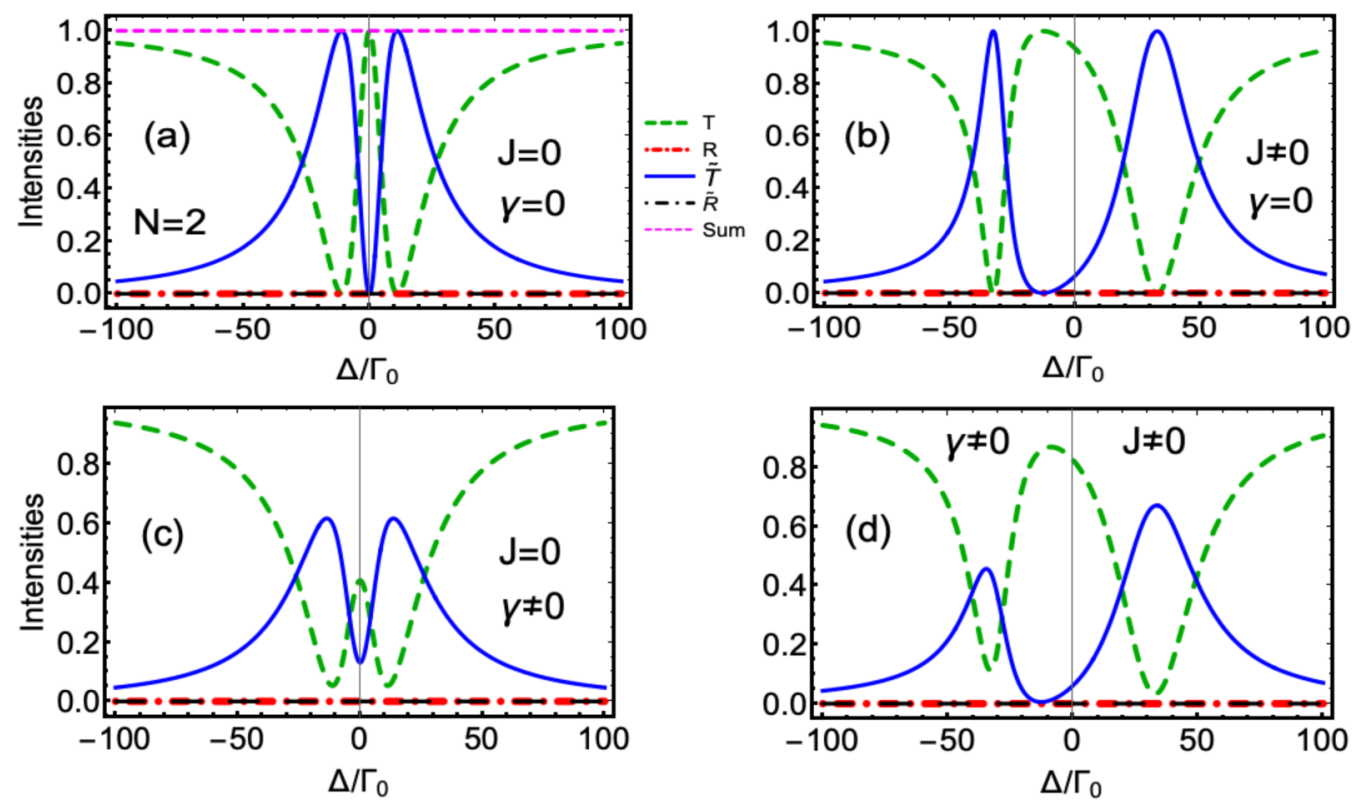

FIG. 4. Single-photon $T, \widetilde{T}, R$, and $\widetilde{R}$ spectra/intensities for two QEs separated by distance $m L=q L=\pi$ as a function of detuning $\Delta$ for a chiral coupling. Four possible cases of DDI between QEs $(J)$ and the spontaneous emission rate $(\gamma)$ are shown. (a) $J=0$ and $\gamma=0$, (b) $\gamma=0$ and emitters are $\lambda_{\mathrm{SP}} / 20$ apart, which, according to Eq. (6), gives rise to $J=23.10 \Gamma_{0}$. (c) $J=0$ and $\gamma=6.86 \Gamma_{0}$. (d) $J=23.10 \Gamma_{0}$ and $\gamma=6.86 \Gamma_{0}$. In all plots $\Gamma=11.03 \Gamma_{0}$. 
(a)

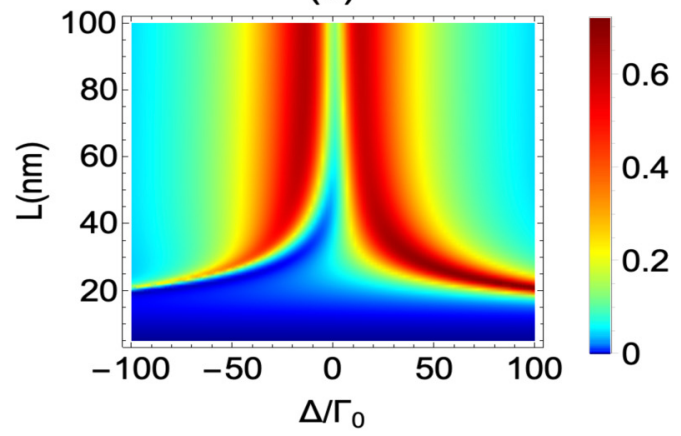

(b)

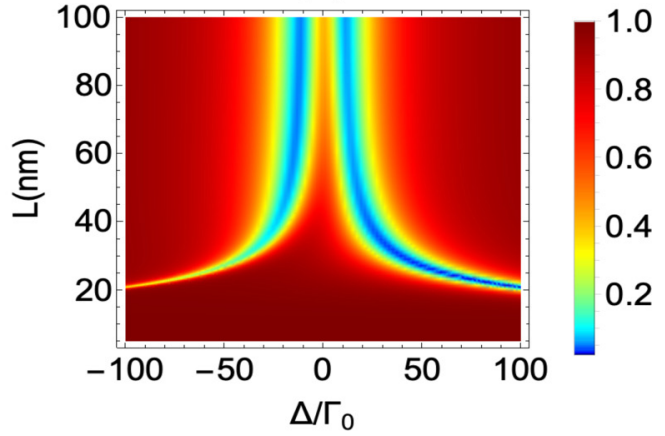

FIG. 5. Density plot showing the single-photon (a) rightward rectification $(\widetilde{T})$ and (b) lower waveguide transmission $(T)$ as a function of detuning $\Delta$ and interemitter separation $L$ for a system of two QEs chirally coupled to a waveguide ladder. The emitter-waveguide coupling is chosen to be $\Gamma=11.03 \Gamma_{0}$ with spontaneous emission rate $\gamma=6.86 \Gamma_{0}$.

not coupled through DDI and spontaneous emission has been disregarded. For this situation, Eq. (15) simplifies to

$$
t_{2}=\frac{\Gamma^{2}-\Delta^{2}}{(\Gamma-i \Delta)^{2}}, \quad \tilde{t}_{2}=\frac{2 i \Gamma \Delta}{(\Gamma-i \Delta)^{2}} .
$$

We note that the term $\Gamma$ appearing in the demonstrator of $t_{2}$ and $\widetilde{t}_{2}$ expressions in Eq. (16) represents an effective DDI between QEs mediated through the waveguides (see also Ref. [36] for a similar argument). And this effective DDI influences the spectral line shape and gives rise to a splitting of peaks in Fig. 4(a). We further notice that, at resonance, there is no rightward rectification but the system shows complete transparency from the bottom waveguide, i.e., $t$ reaches a unit value. On the other hand, it follows from Eq. (16) as well as from Fig. 4(a) that when $\Delta= \pm \Gamma$ the bottom waveguide transmission vanishes, while rightward rectification takes a unit value. Combined, these features generate a frequency separation between both peaks of value $2 \Gamma$. It is worthwhile to point out that even without DDI two QEs can achieve deterministic routing at two different (symmetric) values of detuning, which was not possible otherwise with a single QE.

In the next case, we still neglect the spontaneous emission but consider a finite DDI between the QEs. For this situation Eq. (15) gives

$$
\begin{aligned}
t_{2} & =\frac{-2 \Gamma(\cos \Theta J+\Delta)}{i J^{2}+2 e^{i \theta} J \Gamma+i(\Gamma-i \Delta)}, \\
\tilde{t}_{2} & =\frac{2 i J \Gamma \sin \Theta+i\left(J^{2}+\Gamma^{2}-\Delta^{2}\right)}{i J^{2}+2 e^{i \theta} J \Gamma+i(\Gamma-i \Delta)} .
\end{aligned}
$$

In the above amplitudes, we notice that the presence of DDI results in a nonvanishing value of free propagation phase $\Theta$ which vanished for the $J=0$ case where the photon propagates in a unidirectional/chiral fashion in the waveguides $[25,41]$. Figure 4(b) shows the spectra for this case. We notice that the DDI lifts the symmetry in peak locations of $\widetilde{T}=\left|\widetilde{t}_{2}\right|^{2}$ with a broadened peak on the positive $\Delta$ axis. Rightward rectification approaches a null value at $\Delta=-\cos \Theta J$ while similar to Fig. 4(a) there are two DDI-dependent frequencies where perfect routing is achievable. These frequencies appear at $\Delta= \pm \sqrt{J^{2}+\Gamma^{2}+2 J \Gamma \sin \Theta}$, which are two roots of maximized $\widetilde{T}$. This behavior suggests that by altering the
QE separation we can tune the frequency values where perfect routing is attained.

Subsequently, we take a more realistic scenario in which QEs are allowed to dissipate through spontaneous emission but there is no DDI, i.e., $J=0$ [see Fig. 4(c)]. For this particular case, Eq. (15) yields

$$
\begin{aligned}
& t_{2}=\frac{\gamma^{2}+4 \Gamma^{2}-4 i \gamma \Delta-4 \Delta^{2}}{(\gamma+2 \Gamma-2 i \Delta)^{2}}, \\
& \tilde{t}_{2}=\frac{-4 \Gamma(\gamma-2 i \Delta)}{(\gamma+2 \Gamma-2 i \Delta)^{2}} .
\end{aligned}
$$

As expected, with a nonzero $\gamma$ value, $\tilde{t}_{2}$ reduces drastically and reaches almost $62 \%$. Spontaneous emission also contributes to the widths of the peaks and shifts the peak values slightly away from their previous value of $\pm \Gamma$ [as shown in Fig. 4(a)]. Our parameter choice assumes an overcoupled regime, i.e., $\Gamma>\gamma$. Under this condition, one can develop a partial understanding of intensities analytically. For a small dissipation, i.e., $\Gamma>\gamma$, at resonance, the approximate form of intensities is given by $t_{2} \approx 4 \Gamma^{2} / 4 \Gamma^{2} \rightarrow 1$ and $\tilde{t}_{2} \approx-\gamma / \Gamma \rightarrow$ 0 . We observe a similar tendency of intensities in Fig. 4(c) near $\Delta=0$.

Finally, in Fig. 4(d) we present the intensities when both $J$ and $\gamma$ are nonzero. We notice that due to spontaneous emission symmetry in the peak heights is lost [see Fig. 4(b) for comparison]. This feature, as also pointed out in Ref. [36], is attributed to the energy loss introduced by the atomic dissipation. However, when we compare Figs. 4(c) and 4(d) from the routing perspective, we notice that the rightward rectification reaches $67 \%$, which is $5 \%$ better than the no DDI case and $9 \%$ better than the single-emitter case, though the peak location for the maximum redirection of the photon from bottom to upper waveguide is shifted towards positive detuning. This result clearly indicates that even for $\gamma \neq 0$ DDI opens the possibility of routing efficiency enhancement at one of the spectral peaks.

In Fig. 4 we considered a single value of DDI, i.e., $J=$ $23.10 \Gamma_{0}$ due to the interemitter separation choice of $L=$ $32.75 \mathrm{~nm}$. To see how DDI can influence the routing as a function of $\Delta$, in Fig. 5 we plot port intensities for a range of DDI by varying the $L$ value between 5 and $100 \mathrm{~nm}$. We notice that the DDI considerably changes $\widetilde{T}$ and consequently $T$. 

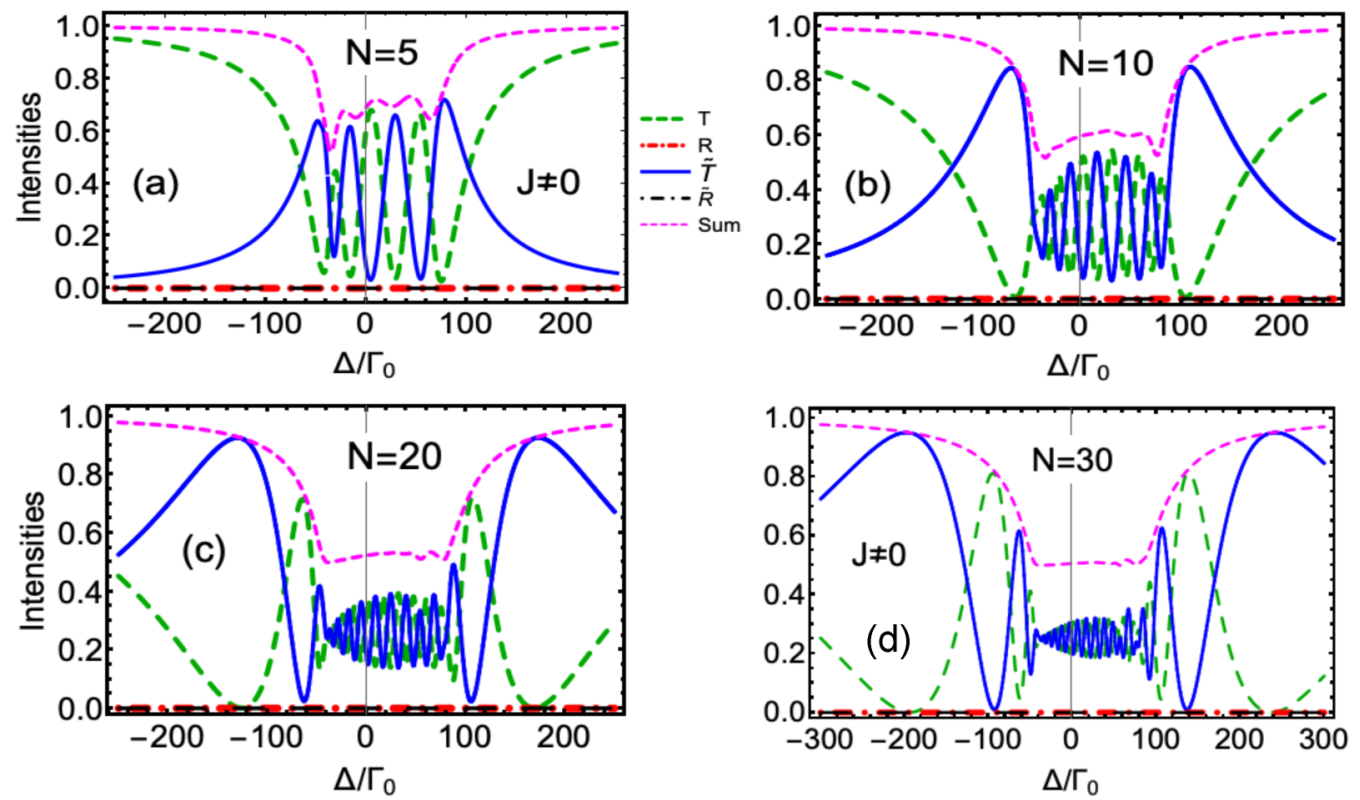

FIG. 6. The single-photon transmission and reflection intensity from the bottom waveguide and the leftward and rightward rectification intensity from the upper waveguide for a chain of (a) 5, (b) 10, (c) 20, and (d) 30 identical dipole-dipole interacting QEs. The separation between any two consecutive emitters in all plots is fixed to $\lambda_{\mathrm{SP}} / 20$ while $\gamma=6.86 \Gamma_{0}$ and $\Gamma=11.03 \Gamma_{0}$.

In particular, above $L \sim 20 \mathrm{~nm}$ we find two regions between $-40 \Gamma_{0} \lesssim \Delta \lesssim 0$ and $0 \lesssim \Delta \lesssim 40 \Gamma_{0}$ where the probability of photon detection at port 3 is at least $60 \%$, i.e., $\widetilde{T} \geqslant 60 \%$. Correspondingly, in the same regions, lower waveguide transmission $T$ remains less than $20 \%$ approximately. Outside these regions the behaviors of $\widetilde{T}$ and $T$ show opposite trends, indicating a progressive deterioration in the photon routing.

\section{Routing improvement due to collective effects of multiple QEs}

Recently it has been reported that the many-emitter waveguide QED setups can outperform single-emitter waveguide QED in performing certain quantum information tasks. For instance, Jones et al. have shown that collective effects arising from a 10-15 QE chain can result in a near-perfect chiral light-matter interaction [42]. Mahmoodian et al. reported that strongly correlated photon emission can be achieved in optically dense emitter ensembles chirally coupled to waveguides even with weak coupling strengths [43]. And Mukhopadhyay and Agarwal have discussed the possibility of achieving perfect transparency in a chain of an even number of nonidentical QEs coupled to a waveguide with separation equal to a halfintegral multiple of resonant wavelength [44].

Motivated by the aforementioned studies, we now discuss the impact of the collective response of multiple QEs on the photon routing scheme. We find that with more than three QEs analytic expressions become intractable; therefore, we show numerical results. In Fig. 6 we present the routing intensities for a moderately sized chain of 30 QEs. From Fig. 6(a) we find that with five QEs both $T=\left|t_{5}\right|^{2}$ and $\widetilde{T}=\left|\widetilde{t}_{5}\right|^{2}$ intensities split into four peaks around resonance. Similar to the two QE cases presented in Fig. 4(d), we find that the DDI results in an asymmetrical spectrum, although with five QEs the highest value of $\widetilde{T}$ can reach as high as $72 \%$ at a far detuned value of $\Delta=85.33 \Gamma_{0}$.

From Figs. 6(b)-6(d) we notice that as the number of QEs increases from 5 to 30 the number of near-resonance peaks in $\widetilde{T}$ (and $T$ ) forms an asymmetric envelope. Additionally, from $N=10$ onward we observe that the $T$ spectra exhibit two maximum peaks that appear on both sides of the envelope. For $N=10,20$, and 30 these peaks appear on the positive detuning axis at $\Delta=108.36 \Gamma_{0}, 173.33 \Gamma_{0}$, and $241.53 \Gamma_{0}$ with respective $\widetilde{T}$ values of $0.850,0.926$, and 0.951 . Interestingly, these peaks, which shift to higher $\Delta$ values with longer emitter chains, not only result in a higher rightward rectification but also result in smaller lower waveguide transmission. Notably, such behavior is contrary to a single waveguide QED case

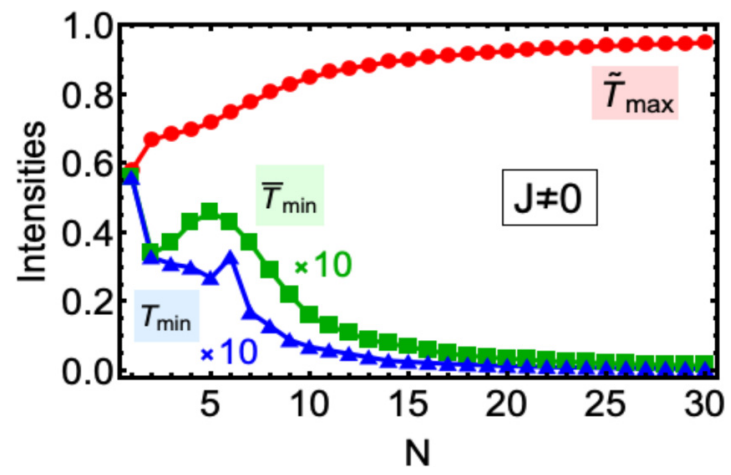

FIG. 7. Routing efficiency enhancement as a function of emitter number $N . \widetilde{T}_{\max }$ represents maximum rightward rectification, $T_{\min }$ displays the minimum value of transmission from the lower waveguide, while $\bar{T}_{\text {min }}$ is the lower waveguide transmission corresponding to the frequency where maximum $\widetilde{T}_{\max }$ has been recorded. Both $T_{\min }$ and $\bar{T}_{\min }$ have been magnified ten times to fit the scale of the plot. The rest of the parameters are the same as used in Fig. 6. 
where a far-off resonant photon transmits the system without interacting with the emitters [25].

To further emphasize the improvement in routing with longer QE chains, in Fig. 7 we report emitter number $N$ dependence of maximum rightward rectification $\widetilde{T}_{\max }$, minimum lower waveguide transmission $T_{\min }$, and lower waveguide transmission $\bar{T}_{\min }$ at the same frequency where $\widetilde{T}_{\max }$ is achieved. We remark that, as $N \longrightarrow 30, \widetilde{T}_{\max }$ reaches $95 \%$ with both $T_{\min }$ and $\bar{T}_{\text {min }}$ diminishing to almost null values. This behavior demonstrates that the collective effects arising from infinitely long-ranged DDI among 30 QEs protect the routing scheme from spontaneous emission loss. Here it is worthwhile to point out that the net loss to the environmental degrees of freedom (nonwaveguide modes) can be calculated from the relation $1-T-\widetilde{T}$ for the present chiral setting [45]. We indicate that this loss takes an almost $5 \%$ value for the $N=30$ case at the $\Delta$ values where maximum $\widetilde{T}$ is observed [for instance, at $\Delta=241.53 \Gamma_{0}$ as shown in Fig. 6(d)].

\section{CONCLUSIONS}

To conclude, we theoretically studied the problem of improving routing efficiency of single photons in a four-port device consisting of a chain of dipole-dipole interacting QEs chirally coupled to a double waveguide ladder in the presence of spontaneous emission. In our paper, the parameter which is used to control the routing efficiency is the interemitter separation or the lattice constant $L$. By changing the value of $L$ we have found that the dipole-dipole interaction between the emitters can be altered, which can then enhance the redirection probability of photons from port 1 to port 3 . For the case of two QEs, we noticed in the absence of spontaneous emission that the strong waveguide mediated interactions (quantified through the parameter $\Gamma$ ) lead to splitting of the spectra into two symmetric peaks. The peak separation depends on the $\Gamma$ value. The inclusion of a DDI opens a direct back-reflection channel even when both waveguides are chiral. We found that this DDI channel causes an asymmetry in the peak heights and shifts the peak locations. For more realistic scenarios, when spontaneous emission is incorporated, it drastically reduces the peak intensities. However, from the routing point of view, we deduced that two QEs with strong DDI (in QDs coupled to Ag nanowire platforms) can enhance the routing efficiency up to $9 \%$ as compared to the single-emitter case when routing was $58 \%$ efficient with the same spontaneous emission loss. For further improvement in the routing scheme, we extended to the many-emitter case and numerically found that for a 30 noisy (i.e., $\gamma \neq 0$ ) $\mathrm{QE}$ chain the collective effects arising due to long-ranged DDI can redirect the photons from bottom to upper waveguide with $95 \%$ efficiency by tuning to far-detuned frequency values. With the current flourishing research activity in light-matter interfaces based on quantum nanophotonics, our results may find applications in designing reliable quantum networks and quantum communication protocols.

The richness of the setup studied in this paper enables us to explore other fascinating questions. For example, in this paper, we have focused on the excitation loss into the modes other than the waveguide modes (spontaneous emission). However, besides spontaneous emission processes, other decoherence mechanisms, namely, pure dephasing and intrinsic waveguide losses, can also have a nontrivial impact on photon routing. However, the inclusion of such processes will either require a full master equation analysis or a substantial modification of present calculations. Therefore, we leave these interesting modifications as a possible future direction of this paper.

\section{ACKNOWLEDGMENTS}

The authors would like to thank Ana Asenjo-Garcia for useful discussions, Yuxiang Zhang for valuable comments on the manuscript and Zibo Wang for suggestions on the numerical code. This work is supported by the Miami University College of Arts and Science and Physics Department start-up funding.
[1] H. J. Kimble, The quantum internet, Nature (London) 453, 1023 (2008).

[2] T. Wilk, S. C. Webster, A. Kuhn, and G. Rempe, Single-atom single-photon quantum interface, Science 317, 488 (2007).

[3] S. Ritter, C. Nölleke, C. Hahn, A. Reiserer, A. Neuzner, M. Uphoff, M. Mücke, E. Figueroa, J. Bochmann, and G. Rempe, An elementary quantum network of single atoms in optical cavities, Nature (London) 484, 195 (2012).

[4] S. E. Harris and Y. Yamamoto, Photon Switching by Quantum Interference, Phys. Rev. Lett. 81, 3611 (1998).

[5] G. S. Agarwal and S. Huang, Optomechanical systems as single-photon routers, Phys. Rev. A 85, 021801(R) (2012).

[6] L. Zhou, L.-P. Yang, Y. Li, and C. P. Sun, Quantum routing of Single Photons with a Cyclic Three-Level System, Phys. Rev. Lett. 111, 103604 (2013).

[7] J. Lu, L. Zhou, L.-M. Kuang, and F. Nori, Single-photon router: Coherent control of multichannel scattering for single photons with quantum interferences, Phys. Rev. A 89, 013805 (2014).
[8] J. Lu, Z. Wang, and L. Zhou, T-shaped single-photon router, Opt. Express 23, 22955 (2015).

[9] W. Qin and F. Nori, Controllable single-photon transport between remote coupled-cavity arrays, Phys. Rev. A 93, 032337 (2016).

[10] M. Ahumada, P. A. Orellana, F. Domínguez-Adame, and A. V. Malyshev, Tunable single-photon quantum router, Phys. Rev. A 99, 033827 (2019).

[11] K. Xia, F. Jelezko, and J. Twamley, Quantum routing of single optical photons with a superconducting flux qubit, Phys. Rev. A 97, 052315 (2018).

[12] T. Aoki, A. S. Parkins, D. J. Alton, C. A. Regal, B. Dayan, E. Ostby, K. J. Vahala, and H. J. Kimble, Efficient Routing of Single Photons by One Atom and a Microtoroidal Cavity, Phys. Rev. Lett. 102, 083601 (2009).

[13] I. Shomroni, S. Rosenblum, Y. Lovsky, O. Bechler, G. Guendelman, and B. Dayan, All-optical routing of single photons by a one-atom switch controlled by a single photon, Science 345, 903 (2014). 
[14] I.-C. Hoi, C. M. Wilson, G. Johansson, T. Palomaki, B. Peropadre, and P. Delsing, Demonstration of a Single-Photon Router in the Microwave Regime, Phys. Rev. Lett. 107, 073601 (2011).

[15] D. Roy, C. M. Wilson, and O. Firstenberg, Colloquium: Strongly interacting photons in one-dimensional continuum, Rev. Mod. Phys. 89, 021001 (2017).

[16] X. Li and L. F. Wei, Designable single-photon quantum routings with atomic mirrors, Phys. Rev. A 92, 063836 (2015).

[17] Y. T. Zhu and W. Z. Jia, Single-photon quantum router in the microwave regime utilizing double superconducting resonators with tunable coupling, Phys. Rev. A 99, 063815 (2019).

[18] D.-C. Yang, M.-T. Cheng, X.-S. Ma, J. Xu, C. Zhu, and X.-S. Huang, Phase-modulated single-photon router, Phys. Rev. A 98, 063809 (2018).

[19] P. Lodahl, S. Mahmoodian, S. Stobbe, A. Rauschenbeutel, P. Schneeweiss, J. Volz, H. Pichler, and P. Zoller, Chiral quantum optics, Nature (London) 541, 473 (2017).

[20] J. Petersen, J. Volz, and A. Rauschenbeutel, Chiral nanophotonic waveguide interface based on spin-orbit interaction of light, Science 346, 67 (2014).

[21] I. M. Mirza and J. C. Schotland, Multiqubit entanglement in bidirectional-chiral-waveguide QED, Phys. Rev. A 94, 012302 (2016).

[22] C. Gonzalez-Ballestero, A. Gonzalez-Tudela, F. J. GarciaVidal, and E. Moreno, Chiral route to spontaneous entanglement generation, Phys. Rev. B 92, 155304 (2015).

[23] I. M. Mirza and J. C. Schotland, Two-photon entanglement in multiqubit bidirectional-waveguide QED, Phys. Rev. A 94, 012309 (2016).

[24] S. Mahmoodian, K. Prindal-Nielsen, I. Söllner, S. Stobbe, and P. Lodahl, Engineering chiral light-matter interaction in photonic crystal waveguides with slow light, Opt. Mater. Express 7, 43 (2017).

[25] I. M. Mirza, J. G. Hoskins, and J. C. Schotland, Chirality, band structure, and localization in waveguide quantum electrodynamics, Phys. Rev. A 96, 053804 (2017).

[26] S. Mahmoodian, G. Calajó, D. E. Chang, K. Hammerer, and A. S. Sørensen, Dynamics of Many-Body Photon Bound States in Chiral Waveguide QED, Phys. Rev. X 10, 031011 (2020).

[27] I. M. Mirza and J. C. Schotland, Influence of disorder on electromagnetically induced transparency in chiral waveguide quantum electrodynamics, JOSA B 35, 1149 (2018).

[28] I. M. Mirza, J. G. Hoskins, and J. C. Schotland, Dimer chains in waveguide quantum electrodynamics, Opt. Commun. 463, 125427 (2020).

[29] I. Söllner, S. Mahmoodian, S. L. Hansen, L. Midolo, A. Javadi, G. Kiršanske, T. Pregnolato, H. El-Ella, E. H. Lee, J. D. Song et al., Deterministic photon-emitter coupling in chiral photonic circuits, Nat. Nanotechnol. 10, 775 (2015).
[30] M.-T. Cheng, X.-S. Ma, J.-Y. Zhang, and B. Wang, Single photon transport in two waveguides chirally coupled by a quantum emitter, Opt. Express 24, 19988 (2016).

[31] C. Gonzalez-Ballestero, E. Moreno, F. J. Garcia-Vidal, and A. Gonzalez-Tudela, Nonreciprocal few-photon routing schemes based on chiral waveguide-emitter couplings, Phys. Rev. A 94, 063817 (2016).

[32] C.-H. Yan, Y. Li, H. Yuan, and L. F. Wei, Targeted photonic routers with chiral photon-atom interactions, Phys. Rev. A 97 023821 (2018).

[33] P. W. Milonni and P. L. Knight, Retardation in the resonant interaction of two identical atoms, Phys. Rev. A 10, 1096 (1974).

[34] J.-T. Shen and S. Fan, Coherent photon transport from spontaneous emission in one-dimensional waveguides, Opt. Lett. 30, 2001 (2005).

[35] G. S. Agarwal, Quantum Optics (Cambridge University, Cambridge, England, 2012).

[36] M.-T. Cheng, J. Xu, and G. S. Agarwal, Waveguide transport mediated by strong coupling with atoms, Phys. Rev. A 95 , 053807 (2017).

[37] R. Schoelkopf and S. Girvin, Wiring up quantum systems, Nature (London) 451, 664 (2008).

[38] J. D. Hood, A. Goban, A. Asenjo-Garcia, M. Lu, S.-P. Yu, D. E. Chang, and H. Kimble, Atom-atom interactions around the band edge of a photonic crystal waveguide, Proc. Natl. Acad. Sci. USA 113, 10507 (2016).

[39] A. Akimov, A. Mukherjee, C. Yu, D. Chang, A. Zibrov, P. Hemmer, H. Park, and M. Lukin, Generation of single optical plasmons in metallic nanowires coupled to quantum dots, Nature (London) 450, 402 (2007).

[40] D. E. Chang, A. S. Sørensen, P. R. Hemmer, and M. D. Lukin, Quantum Optics with Surface Plasmons, Phys. Rev. Lett. 97, 053002 (2006).

[41] I. M. Mirza, S. van Enk, and H. Kimble, Single-photon timedependent spectra in coupled cavity arrays, J. Opt. Soc. Amer. B 30, 2640 (2013).

[42] R. Jones, G. Buonaiuto, B. Lang, I. Lesanovsky, and B. Olmos, Collectively Enhanced Chiral Photon Emission from an Atomic Array Near a Nanofiber, Phys. Rev. Lett. 124, 093601 (2020).

[43] S. Mahmoodian, M. Čepulkovskis, S. Das, P. Lodahl, K. Hammerer, and A. S. Sørensen, Strongly Correlated Photon Transport in Waveguide Quantum Electrodynamics with Weakly Coupled Emitters, Phys. Rev. Lett. 121, 143601 (2018).

[44] D. Mukhopadhyay and G. S. Agarwal, Transparency in a periodic chain of quantum emitters strongly coupled to a waveguide, Phys. Rev. A 101, 063814 (2020).

[45] A. Asenjo-Garcia, M. Moreno-Cardoner, A. Albrecht, H. J. Kimble, and D. E. Chang, Exponential Improvement in Photon Storage Fidelities Using Subradiance and "Selective Radiance" in Atomic Arrays, Phys. Rev. X 7, 031024 (2017). 